Rev. Biol. Neotrop. 9(1): 28-37. 2012

\title{
Vão do Paranã (GO) como área chave para conservação de MACROINVERTEBRADOS BENTÔNICOS
}

\author{
Silvia Leitão Dutra \\ Universidade Federal do Tocantins, Ciências Naturais. E-mail: silviad@uft.edu.br
}

\author{
Victor Lemes LaNDeiro \\ Universidade Federal de Mato Grosso, Departamento de Botânica e Ecologia. \\ E-mail: vllandeiro@gmail.com
}

Leandro Gonçalves Oliveira

Universidade Federal de Goiás, Laboratório de Meio Ambiente e Gerenciamento de Recursos Hídricos (LAMARH). E-mail: lego@icb.ufg.br

\begin{abstract}
Resumo: Foi feita uma indicação de área prioritária para conservação da biota de invertebrados aquáticos na bacia hidrográfica do rio Paranã-GO, utilizando a comunidade de macroinvertebrados bentônicos como ferramenta de bioindicação. Os invertebrados foram coletados pela metodologia de avaliação rápida, utilizando redes manuais de abertura de malha de $2 \mathrm{~mm}$ durante 15 minutos de coleta ativa, em ambientes de correnteza com substrato pedregoso. A escolha da área foi feita seguindo três critérios: 1- Raridade do táxon; 2- Representatividade e complementaridade dos pontos amostrados e 3- Conectividade. A área selecionada inclui trechos de rio com diferentes características hidrológicas, que refletem as diferentes características de composição da biota. A área prioritária para conservação proposta neste trabalho inclui três microbacias: as dos rios São Bartolomeu, Corrente e São Mateus. A área indicada é uma região de expansão de atividades antrópicas e não está atualmente protegida por unidades de conservação federais ou estaduais. As informações geradas nesse trabalho podem subsidiar uma gestão mais eficiente para a conservação da biota aquática da bacia do rio Paranã.
\end{abstract}

Palavras-Chave: Complementaridade, raridade, conectividade, invertebrados aquáticos, avaliação rápida.

Abstract: In this study we suggested the indication of the Paranã River watershed, Goiás, as a priority area for conservation of aquatic invertebrates. It was made based on the use of the benthic macroinvertebrate community as a bioindication tool. The invertebrates were collected by rapid assessment methodology, using hand nets of mesh size of $2 \mathrm{~mm}$ during 15 minutes of active sampling in current environments with rubble substrate. The choice of the area was made following three criteria: 1- Rarity of the taxon; 2-Representation and complementarity of sampled points; 3-Connectivity. The selected area includes stretches of river with different hydrological characteristics, which are reflected in the different compositional characteristics of the biota. The priority area for conservation proposed in this paper includes three watersheds: the rivers São Bartolomeu, Corrente and São Mateus. The indicated area is not currently protected by federal or state units of conservation and also this region suffer current expansion of anthropic activities. The information resulted from this study can serve as support for a more efficient management of the conservation of the aquatic biota of the Paranã River.

KeY WoRds: Rarity, complementarity, connectivity, aquatic invertebrates, fast evaluation.

\section{INTRODUÇÃO}

O rias para conservação biológica tem como objetivo assegurar a persistência de espécies, comunidades e paisagens num espaço geográfico específi- co (Araújo, 1998). A eficiência desse processo é dependente da qualidade das informações biológicas disponíveis, que no caso, pode ser um dos problemas mais sérios, principalmente em sistemas neotropicais, que apresenta grandes áreas pouco conhecidas ou não estudadas, gerando uma heterogeneidade no grau de conhecimento sobre 
os táxons, habitats e biomas. Os táxons menos conhecidos quanto à distribuição, abundância ou requerimentos ecológicos acabam sendo negligenciados e simplesmente não são incluídos nas medidas de conservação. Essa ignorância leva a escolha de estratégias de conservação equivocadas que não garantem a manutenção de muitas espécies no ambiente, podendo causar até mesmo a extinção de espécies ainda não catalogadas pela ciência (Brandon et al., 2005; De Marco \& Vianna, 2005; Diniz-Filho et al., 2010). Essa escassez de informações biológicas também não tem permitido o estabelecimento de prioridades e de ações práticas para a conservação da enorme biodiversidade brasileira (De Marco \& Vianna, 2005), especialmente dos invertebrados. Estima-se que das oito milhões de espécies de insetos existentes no mundo (Mora et al., 2011), cerca de um milhão está presente no Brasil (Lewinsohn et al., 2005). Apesar da imensa riqueza biológica, apenas uma ínfima parte foi descrita e depositada em coleções zoológicas brasileiras (Zaher \& Young, 2003). Este fato torna-se ainda mais alarmante para a biota aquática que é ainda menos conhecida (Strayer, 2006).

Os invertebrados aquáticos desempenham um papel ecológico fundamental em ecossistemas lóticos, em razão de sua grande contribuição no fluxo de matéria e energia (Dudgeon, 2008). Eles favorecem a ciclagem de nutrientes através da fragmentação de matéria orgânica e disponibilização de nutrientes para outros organismos da cadeia alimentar (Bispo et al., 2001; Vannote et al., 1980). Ainda, os macroinvertebrados aquáticos são considerados bons indicadores naturais da qualidade ambiental (Miserendino \& Pissolón, 2001) e são amplamente utilizados em estudos de biomonitoramento (Junqueira et al., 2000), de avaliação da qualidade da água e da integridade ambiental (Silveira et al., 2005).

Diante deste contexto destacamos a importância da conservação da Região do Vão do Rio Paranã, GO, inserida no bioma Cerrado, considerado como hotspot de biodiversidade (Myers et al., 2000). Estudos limnológicos são necessários nesta bacia hidrográfica que foi classificada como de pouco ou nenhum conhecimento biológico sobre fauna e flora e como de alta prioridade para conservação da biota aquática (área 217), de acordo com as indicações do Workshop "Ações prioritárias para conservação da biodiversidade do Cerrado e Pantanal" (MMA, 1999). Poucos estudos realizados destacaram a importância dos levantamentos da comunidade bentônica nesta bacia, em função da alta frequência de novos registros de ocorrência de gêneros para o estado de Goiás (Dutra, 2006; Martins-Silva et. all, 2008; Barbosa et. all, 2011).

O Vão do Paranã está localizado em uma região de Goiás que ainda apresenta boas condições de qualidade ambiental (Ferreira, 2008). Tal estado é confirmado pela grande variedade de táxons na comunidade bentônica coletados em sua bacia hidrográfica, facilitando dessa maneira a indicação de prioridade para conservação pela relação das variações de composição de espécies ligadas ao ambiente natural e não pelas atividades antrópicas (McGeoch 1998).

A proposta desse trabalho foi indicar uma área prioritária para conservação de macroinvertebrados bentônicos que possa garantir a sua representação máxima na região do Vão do Rio Paranã, a partir de um levantamento da biodiversidade realizado nesta bacia.

\section{Material e Métodos}

O Vão do Paranã está inserido no bioma Cerrado e faz parte da bacia hidrográfica Araguaia-Tocantis, localizada na região Centro-Oeste do Brasil. O clima da região é do tipo $\mathrm{CW}$ de acordo com a classificação de Köppen, o que indica um clima com temperaturas elevadas (média anual de $25,6^{\circ} \mathrm{C}$ ), com chuvas no verão (outubro-março) e seca no inverno (abril-setembro) (Nimer, 1979).

Foram amostrados 16 pontos em agosto de 2003 no Vão do Paranã (Tab. 1). Esses pontos foram classificados de acordo com a ordem de classificação hidrológica proposta por Strahler (1957). Foi aplicada a metodologia de avaliação rápida de qualidade de água, amostrando o máximo de locais com menor número de réplicas de habitats (Barbour et al., 1999; Buss et al., 2003; Rosenberg \& Resch, 1993).

Tabela 1 Localização e classificação da ordem hidrológica dos pontos amostrados no Vão do Paranã no ano de 2003.

\begin{tabular}{|c|c|c|c|c|}
\hline \multicolumn{2}{|c|}{ Ponto Amostral } & \multicolumn{2}{|c|}{ Coordenadas } & \multirow{2}{*}{$\frac{\text { Ordem Hidrológica }}{2}$} \\
\hline $\mathbf{A}$ & Jataí & 47W 27' 43" & 15S 06' 58" & \\
\hline B & Água Fria & 47W 27' 41" & 15S 07' 08" & 3 \\
\hline C & São Pedro & 47W 23' 38" & 14S 45' 52" & 2 \\
\hline D & Extrema & 47W 17' 52" & 14S 39' $27^{\prime \prime}$ & 3 \\
\hline $\mathbf{E}$ & Ribeirão & 47W 16' 44" & 14S 30' 43" & 2 \\
\hline $\mathbf{F}$ & Faria & 47W 15' 58" & 14S 27' 12 '" & 3 \\
\hline G & Paranã & 47W 06' 05" & 14S 27' 21 ' & 4 \\
\hline H & Macacos & $47 \mathrm{~W} 00^{\prime} 20^{\prime \prime}$ & $14 S 27^{\prime} 42^{\prime \prime}$ & 4 \\
\hline $\mathbf{I}$ & Corrente & 46W 55' 58" & 14S 18' 19" & 5 \\
\hline $\mathbf{J}$ & São Bartolomeu & 47W 07' 33" & $14 S 05^{\prime} 15^{\prime \prime}$ & 4 \\
\hline $\mathbf{L}$ & Forquilha & 46W 58' 58" & 13S 57' 43" & 2 \\
\hline M & Paranã & 46W 50' 25" & 14S 02' 50" & 5 \\
\hline $\mathbf{N}$ & Água Quente & 46W 38' 06" & 13S 56' 00" & 4 \\
\hline $\mathbf{0}$ & São Mateus & 46W 38' 58" & 13S 48' 43" & 4 \\
\hline $\mathbf{P}$ & Morcego & 46W 52' 23" & 13S 43' $11 "$ & 2 \\
\hline $\mathbf{Q}$ & Paranã & 46W 47' 49" & 13540 ' $10 "$ & 6 \\
\hline
\end{tabular}


A coleta dos macroinvertebrados bentônicos foi padronizada por tempo de amostragem em cada um dos pontos: 15 minutos em ambientes de correnteza e substrato pedregoso. Foram utilizadas redes manuais, de abertura de malha de $2 \mathrm{~mm}$, para recolher o substrato. Com uma pinça entomológica, apenas os macroinvertebrados eram coletados e imediatamente fixados em formol 5\%. O material biológico foi identificado utilizando chaves especializadas (Dominguez et al., 2001; Merritt \& Cummins, 1996; Wiggins, 1977). Os organismos foram conservados em álcool $80 \%$ e depositados na coleção do Laboratório de Limnologia da Universidade Federal de Goiás.

Para avaliar a influencia da classificação hidrológica sobre a riqueza observada foi utilizada a correlação de Pearson. Para avaliar a similaridade da composição de espécies entre as comunidades das unidades amostrais foi feita uma Análise de Correspondência com remoção do efeito do arco (Detrended Correspondence Analysis, DCA). Os pontos de amostragem foram considerados como unidades amostrais para ambas as análises $(n=16)$.

A avaliação para seleção de áreas prioritárias para conservação foi orientada por três critérios, que estão descritos a seguir: (1) Raridade, (2) Representatividade e (3) Conectividade.

1. Raridade. A distribuição dos táxons no meio ambiente não é uniforme, mas também não ocorre ao acaso. Ela é influenciada constantemente por inúmeros fatores históricos (e.g. a capacidade das espécies em chegarem a um determinado local), bióticos e abióticos. Tais fatores determinam a distribuição, a riqueza, e a abundância dos indivíduos ao longo do tempo e do espaço (Lambers et al., 1998; Lomolino \& Perault, 2004; Lortie et al., 2004; Ulrich, 2008). Desta maneira, a raridade se torna um conceito útil na biologia da conservação, por fornecer uma aproximação do grau de vulnerabilidade ou ameaça de extinção (Arita, 1993). Além disso, a raridade contempla também os táxons endêmicos que apresentam área de distribuição restrita, com histórias filogenéticas únicas e insubstituíveis (Harold \& Randall, 1994). Por isso, a raridade tem sido um critério comumente usado em trabalhos de indicação de áreas prioritárias para conservação (Myers et al, 2000). Entre os organismos aquáticos, a raridade e o endemismo podem ser ainda maiores, principalmente para espécies que possuem dispersão limitada e que não resistem à dessecação (Strayer, 2006).

Foram considerados como táxons raros os amostrados em apenas um ou dois pontos de coleta. Os primeiros pontos escolhidos foram os que apresentaram o maior número de táxons raros.

2. Representatividade. A descrição da biodiversidade pode ser feita através da quantificação de táxons (Gaston, 1996). Esta riqueza taxonômica reflete a probabilidade de serem representadas importantes características da co- munidade biológica como a variabilidade genética (Myers, 1988; Myers, 1990; Vane-Wright et al., 1991), de níveis tróficos ou "guildas" (Cousins, 1991), morfológica e ecológica (Kareiva \& Marvier, 2003), além da quantidade de habitats, ecossistemas e paisagens da área estudada (Buckley \& Forbes, 1978). O grau de representação de uma área ou de um conjunto de áreas para incluir a totalidade dos atributos encontrados (espécies ou paisagens) (Vane-Wright, 1991) deve também ser considerado para assegurar a maior representatividade desta comunidade (Vane-Wright, 1991; Myers, 1988; Myers, 1990).

No presente estudo foram identificados os pontos que (i) melhor representassem a biota da bacia hidrográfica e (ii) os pontos com biota complementar, ou seja, mais diferenciada. Isto foi feito observando-se a similaridade entre os pontos através do gráfico da DCA (Fig. 1).

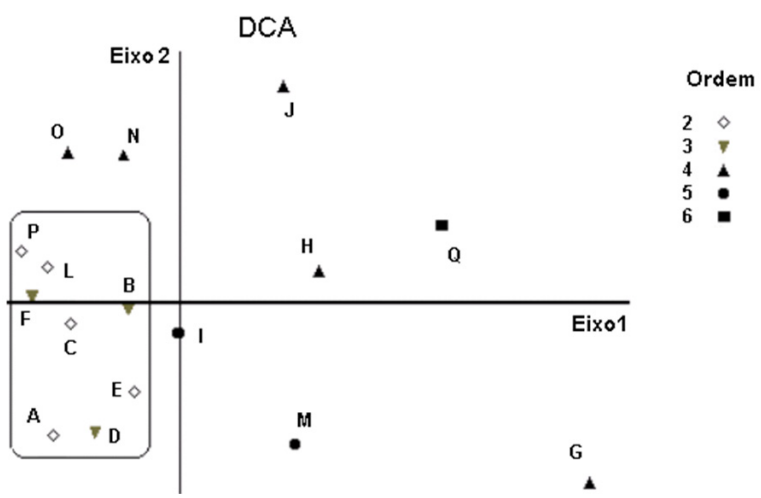

Fig. 1 - Ordenação de pontos de coleta de invertebrados bentônicos, através de DCA com dados bióticos de abundância, explicação no eixo $1=67,9 \%$ e eixo $2=8,1 \%$

3. Conectividade. A comunidade de invertebrados é também regulada pela estrutura e qualidade de todo o sistema lótico: qualidade da água de seus afluentes, pela qualidade de sua mata ripária e pelo grau de conectividade dentro do próprio corpo d'água (Junqueira et al., 2000; Pringle, 2001). Assim, as ações de conservação devem ser propostas dentro dos domínios das microbacias ou das bacias hidrográficas para auxiliar na manutenção natural dos pulsos de inundação, do fluxo de energia e matéria dentro do rio e permitir também a dispersão de organismos aquáticos ao longo do curso do rio. (Karr \& Chu, 1999; Looy et al., 2006; Linke et al.,2007). A indicação da área prioritária para conservação foi estendida às nascentes das microbacias selecionadas para contemplar o critério de conectividade das áreas.

\section{Resultados}

Foram coletados, nos 16 pontos (Tab. 1 e Fig. 2), 3.094 organismos que representaram 54 táxons, sendo $98 \%$ da amostra constituída por insetos (Tab. 2). Os pontos com maior riqueza (S) taxonômica observada foram: ponto N $(S=24)$ 
com predominância de Anacroneuria (Plecoptera) com 43 indivíduos coletados, Oecetis (Trichoptera)(35 indivíduos) e Elmidae (Coleoptera)(32 indivíduos); ponto I $(\mathrm{S}=22)$ apresentou a predominância de dípteros Chironomidae (547 indivíduos) e Simuliidae (344 indivíduos), seguido de Smicridea (Tichoptera) (53 indivíduos), NeedhameIla (Ephemetoptera)(21 indivíduos), Leptohyphes
(Ephemetoptera) (19 indivíduos) e Anacroneuria (4 indivíduos); os pontos $\mathrm{O}, \mathrm{M}$ e $\mathrm{C}$ apresentaram boa representatividade, cada um deles com 21 taxa, com predominância de Farrodes (Ephemeroptera) (38 indivíduos), Leentvaaria (Ephemeroptera) (38 indivíduos) e Chimarra (Trichoptera) (45 indivíduos), respectivamente (Tab. 2).

Tabela 2 Lista de táxons, abundância e riqueza de macroinvertebrados bentônicos amostrados no período de seca (agosto) no Vão do Paranã no ano de 2003.

\begin{tabular}{|c|c|c|c|c|c|c|c|c|c|c|c|c|c|c|c|c|c|}
\hline & $\mathbf{A}$ & B & C & D & $\mathbf{E}$ & $\mathbf{F}$ & $\mathbf{G}$ & $\mathbf{H}$ & $\mathbf{I}$ & $\mathbf{J}$ & $\mathbf{L}$ & $\mathbf{M}$ & $\mathbf{N}$ & 0 & $\mathbf{P}$ & $\mathbf{Q}$ & $\begin{array}{c}\text { Táxons } \\
\text { raros }\end{array}$ \\
\hline \multicolumn{18}{|l|}{ DIPTERA } \\
\hline Ceratopogonidae & 0 & 0 & 0 & 0 & 0 & 0 & 0 & 0 & 0 & 0 & 0 & 0 & 0 & 2 & 0 & 1 & $\mathbf{x}$ \\
\hline Chironomidae & 32 & 16 & 26 & 8 & 33 & 6 & 0 & 8 & 547 & 5 & 35 & 10 & 3 & 3 & 9 & 2 & \\
\hline Simuliidae & 12 & 0 & 0 & 4 & 0 & 4 & 0 & 0 & 344 & 0 & 6 & 2 & 0 & 1 & 3 & 0 & \\
\hline Stratiomyidae & 0 & 0 & 0 & 0 & 0 & 0 & 0 & 0 & 0 & 0 & 0 & 0 & 0 & 2 & 0 & 0 & $\mathbf{x}$ \\
\hline Tipulidae & 0 & 3 & 0 & 0 & 0 & 0 & 0 & 1 & 0 & 2 & 0 & 1 & 0 & 0 & 0 & 0 & \\
\hline \multicolumn{18}{|l|}{ EPHEMEROPTERA } \\
\hline \multicolumn{18}{|l|}{ Baetidae } \\
\hline Baetodes & 0 & 0 & 1 & 0 & 0 & 0 & 0 & 0 & 0 & 0 & 6 & 0 & 0 & 0 & 6 & 0 & \\
\hline Camelobaetidius & 0 & 1 & 1 & 0 & 1 & 1 & 0 & 5 & 1 & 0 & 0 & 0 & 1 & 0 & 2 & 0 & \\
\hline Cloeodes & 0 & 0 & 0 & 2 & 1 & 2 & 0 & 10 & 18 & 13 & 1 & 4 & 2 & 3 & 3 & 13 & \\
\hline Prebaetodes & 0 & 0 & 0 & 0 & 0 & 0 & 0 & 0 & 0 & 0 & 0 & 1 & 2 & 0 & 0 & 0 & $\mathbf{x}$ \\
\hline \multicolumn{18}{|l|}{ Leptophlebiidae } \\
\hline Farrodes & 14 & 7 & 3 & 6 & 1 & 14 & 0 & 0 & 8 & 16 & 1 & 2 & 13 & 38 & 45 & 0 & \\
\hline Hagenulopsis & 0 & 0 & 0 & 0 & 4 & 3 & 0 & 0 & 0 & 1 & 0 & 0 & 0 & 4 & 2 & 0 & \\
\hline Hermanella & 0 & 0 & 0 & 0 & 0 & 0 & 0 & 0 & 0 & 0 & 0 & 0 & 1 & 0 & 0 & 0 & $\mathbf{x}$ \\
\hline Leentvaaria & 42 & 10 & 0 & 3 & 12 & 2 & 0 & 6 & 6 & 0 & 0 & 38 & 0 & 0 & 0 & 0 & \\
\hline Needhamella & 0 & 1 & 0 & 0 & 10 & 0 & 0 & 0 & 41 & 0 & 0 & 11 & 0 & 0 & 0 & 0 & \\
\hline Thraulodes & 2 & 0 & 12 & 1 & 0 & 1 & 0 & 0 & 0 & 0 & 0 & 0 & 0 & 0 & 0 & 0 & \\
\hline Traverella & 0 & 0 & 0 & 0 & 0 & 0 & 0 & 0 & 7 & 0 & 0 & 0 & 0 & 0 & 0 & 0 & $\mathbf{x}$ \\
\hline \multicolumn{18}{|l|}{ Leptohyphidae } \\
\hline Leptohyphes & 4 & 5 & 4 & 4 & 0 & 6 & 1 & 5 & 19 & 1 & 7 & 10 & 3 & 3 & 30 & 1 & \\
\hline Leptohyphodes & 0 & 0 & 1 & 0 & 0 & 0 & 0 & 0 & 0 & 0 & 0 & 0 & 0 & 2 & 0 & 0 & $\mathbf{x}$ \\
\hline Tricorythodes & 0 & 0 & 0 & 0 & 0 & 0 & 0 & 0 & 0 & 0 & 0 & 0 & 0 & 3 & 0 & 0 & $\mathbf{x}$ \\
\hline Tricorythopsis & 0 & 0 & 0 & 0 & 0 & 0 & 0 & 5 & 0 & 0 & 0 & 1 & 1 & 0 & 0 & 0 & \\
\hline \multicolumn{18}{|l|}{ Polymitarcyidae } \\
\hline Campsurus & 0 & 0 & 1 & 0 & 0 & 0 & 1 & 0 & 0 & 0 & 0 & 4 & 0 & 0 & 0 & 0 & \\
\hline \multicolumn{18}{|l|}{ PLECOPTERA } \\
\hline \multicolumn{18}{|l|}{ Perlidae } \\
\hline Anacroneuria & 4 & 5 & 15 & 1 & 2 & 6 & 0 & 0 & 4 & 0 & 10 & 0 & 43 & 4 & 13 & 0 & \\
\hline \multicolumn{18}{|l|}{ TRICHOPTERA } \\
\hline \multicolumn{18}{|l|}{ Leptoceridae } \\
\hline Oecetis & 0 & 0 & 0 & 0 & 0 & 0 & 0 & 0 & 0 & 0 & 0 & 0 & 35 & 0 & 0 & 1 & $\mathbf{x}$ \\
\hline \multicolumn{18}{|l|}{ Polycentropodidae } \\
\hline Cyrnellus & 0 & 0 & 0 & 0 & 0 & 0 & 0 & 1 & 0 & 1 & 1 & 0 & 0 & 0 & 0 & 0 & \\
\hline
\end{tabular}


Tabela 2. Continuação. Lista de táxons, abundância e riqueza de macroinvertebrados bentônicos amostrados no período de seca (agosto) no Vão do Paranã no ano de 2003.

\section{$\begin{array}{lllllllllllllllll}\text { A } & \text { B } & \text { C } & \text { D } & \text { E } & \text { F } & \text { G } & \text { H } & \text { I } & \text { J } & \text { L } & \text { M } & \mathbf{N} & \mathbf{O} & \mathbf{P} & \mathbf{Q} & \begin{array}{c}\text { Táxons } \\ \text { raros }\end{array}\end{array}$}

\begin{tabular}{|c|c|c|c|c|c|c|c|c|c|c|c|c|c|c|c|c|c|}
\hline \multicolumn{18}{|l|}{ Hydrobiosidae } \\
\hline Atopsyche & 0 & 0 & 1 & 0 & 0 & 0 & 0 & 0 & 0 & 0 & 0 & 0 & 0 & 0 & 0 & 0 & $\mathbf{x}$ \\
\hline \multicolumn{18}{|l|}{ Helicopsychidae } \\
\hline Helicopsyche & 0 & 0 & 0 & 0 & 1 & 0 & 0 & 0 & 0 & 8 & 0 & 0 & 1 & 2 & 0 & 0 & \\
\hline Odontoceridae & 0 & 0 & 1 & 0 & 0 & 0 & 0 & 0 & 0 & 0 & 0 & 0 & 0 & 0 & 0 & 0 & $\mathbf{x}$ \\
\hline \multicolumn{18}{|l|}{ Hydroptilidae } \\
\hline Dicaminus & 0 & 0 & 0 & 0 & 2 & 0 & 0 & 0 & 0 & 0 & 0 & 0 & 0 & 0 & 0 & 0 & $\mathbf{x}$ \\
\hline Hydroptila & 0 & 0 & 0 & 0 & 0 & 0 & 0 & 0 & 15 & 0 & 0 & 0 & 0 & 0 & 0 & 1 & $\mathbf{x}$ \\
\hline Oxyethira & 0 & 0 & 1 & 0 & 0 & 0 & 0 & 0 & 0 & 1 & 0 & 0 & 0 & 0 & 0 & 0 & $\mathbf{x}$ \\
\hline \multicolumn{17}{|l|}{ Philopotamidae } & \\
\hline Chimarra & 120 & 58 & 45 & 50 & 36 & 19 & 0 & 0 & 23 & 1 & 92 & 6 & 12 & 20 & 4 & 0 & \\
\hline \multicolumn{17}{|l|}{ Hydropsychidae } & \\
\hline Leptonema & 12 & 12 & 3 & 4 & 8 & 5 & 0 & 10 & 0 & 0 & 7 & 0 & 1 & 2 & 11 & 0 & \\
\hline Macronema & 22 & 0 & 0 & 5 & 3 & 0 & 0 & 0 & 1 & 0 & 0 & 4 & 0 & 0 & 0 & 0 & \\
\hline Smicridea & 12 & 4 & 4 & 10 & 6 & 4 & 0 & 24 & 53 & 0 & 7 & 36 & 8 & 6 & 3 & 0 & \\
\hline Synoestropsis & 0 & 0 & 0 & 0 & 0 & 0 & 0 & 0 & 0 & 0 & 0 & 16 & 0 & 0 & 0 & 0 & \\
\hline
\end{tabular}

Glossosomatidae

Protoptila

MEGALOPTERA

Corydalidae

COLEOPTERA

Dytiscidae

Elmidae

Gyrinidae

Psephenidae

ODONATA

Calopterygidae

Coenagrionidae

Gomphidae

Libellulidae

HETEROPTERA

Belostomatidae

Corixidae

Naucoridae

LEPIDOPTERA

ACARINA

PLANÁRIA

GASTROPODA

$\begin{array}{ll}0 & 0 \\ 2 & 4\end{array}$

0

$\begin{array}{lll}0 & 0 & 0\end{array}$

$\begin{array}{ll}0 & 1\end{array}$

00

0

00

$$
00
$$

$\begin{array}{llllllllllllllll}0 & 2 & 2 & 0 & 0 & 0 & 0 & 4 & 1 & 2 & 2 & 0 & 4 & 2 & 0 & 1 \\ 7 & 2 & 3 & 3 & 9 & 1 & 0 & 0 & 1 & 0 & 2 & 2 & 1 & 2 & 1 & 3 \\ 0 & 0 & 0 & 0 & 1 & 0 & 1 & 0 & 0 & 0 & 0 & 0 & 0 & 0 & 0 & 2 \\ 0 & 1 & 1 & 0 & 0 & 0 & 0 & 0 & 36 & 3 & 1 & 2 & 1 & 2 & 0 & 4\end{array}$

CRUSTACEA

BIVALVA

Riqueza

observada

Riqueza de

$\begin{array}{ccccccccccccccccccc}0 & 0 & 5 & 1 & 7 & 3 & 0 & 0 & 1 & 1 & 0 & 1 & 1 & 0 & 0 & 1 & \\ 0 & 0 & 0 & 0 & 0 & 0 & 0 & 0 & 0 & 0 & 0 & 0 & 2 & 0 & 0 & 0 & \mathbf{x} \\ 1 & 3 & 5 & 0 & 0 & 3 & 0 & 1 & 0 & 1 & 2 & 0 & 1 & 8 & 0 & 0 & \\ 0 & 0 & 0 & 0 & 0 & 0 & 0 & 0 & 13 & 0 & 0 & 0 & 0 & 0 & 0 & 0 & \mathbf{x} \\ 0 & 0 & 0 & 0 & 0 & 2 & 0 & 0 & 2 & 0 & 1 & 0 & 0 & 1 & 0 & 0 & \\ 0 & 0 & 0 & 0 & 0 & 0 & 0 & 0 & 3 & 0 & 0 & 0 & 1 & 0 & 1 & 0 & \\ 0 & 1 & 0 & 0 & 0 & 0 & 0 & 35 & 0 & 2 & 0 & 2 & 0 & 0 & 0 & 0 & \\ 0 & 0 & 0 & 0 & 0 & 0 & 0 & 0 & 0 & 1 & 0 & 0 & 0 & 0 & 0 & 0 & \mathbf{x} \\ 0 & 0 & 0 & 0 & 0 & 0 & 0 & 4 & 0 & 0 & 0 & 3 & 0 & 0 & 0 & 2 & \\ 15 & 18 & 21 & 15 & 19 & 20 & 3 & 15 & 22 & 19 & 18 & 21 & 24 & 21 & 16 & 13 & \\ 0 & 0 & 4 & 0 & 1 & 1 & 0 & 0 & 3 & 4 & 0 & 2 & 5 & 4 & 0 & 4 & \end{array}$


A riqueza observada não foi correlacionada com a classificação hidrológica do rio (Pearson $=-0.08357$ ), mas foi relacionada com o eixo 1 da DCA (explicação de 67,9\% para o eixo 1 e de $8,1 \%$ para o eixo 2) (Fig. 1). Há uma diferenciação entre dois grupos distintos na DCA: O grupo formado por pontos de baixa classificação hidrológica ( $2^{a}$ à $3^{a}$ ordens) foi destacado no gráfico, pois apresentam grande similaridade quanto a sua composição. Os outros pontos apresentam maior ordem hidrológica (4a à $6^{a}$ ordens) e foram menos similares entre si, formando um grupo mais disperso no gráfico (Fig. 1).

$\mathrm{O}$ ponto com maior riqueza de táxons raros foi o ponto $\mathrm{N}$ que apresentou cinco táxons (Prebaetodes, Hermanella, Oecetis, Gyrinidae e Corixidae). Outros quatro pontos ( $\mathrm{C}, \mathrm{J}, \mathrm{O}$ e Q) apresentaram riqueza de quatro táxons raros (Tab. 2).

O ponto I apresentou a maior similaridade entre os pontos e sua posição central no gráfico da DCA (Fig. 1) indica que ele representou a biota mais comum da bacia hidrográfica. Seguindo-se o princípio da complementaridade, os pontos de menor similaridade com os demais foram $G, O, J$ e Q. O ponto $\mathrm{G}$ foi excluído por apresentar apenas táxons comuns em vários pontos da bacia hidrográfica (Leptohyphes, Campsurus, Gomphidae). Os outros pontos foram mantidos devido aos táxons de baixa constância: Stratiomyidae, Tri- corythodes e Leptohyphodes, presentes no ponto O; Oxyethira, Psephenidae, Cyrnellus, Dytiscidae e crustáceos encontrados no ponto J; além de Ceratopogonidae e Oecetis presentes no ponto Q.

Dos seis pontos localizados inicialmente $(\mathrm{N}$, C, J, O, Q e I), cinco encontram-se na porção média do Rio e apenas o ponto $C$ encontra-se a montante na porção superior (Fig. 2). A biota presente neste ponto é muito similar à biota presente em todos os outros córregos de baixa ordem nesta bacia (Fig. 1). O ponto C foi excluído da área indicada, pois a biota deste ponto também estaria bem representada através de outros dois pontos mais próximos, o L e $P$.

A partir dos cinco pontos iniciais $(\mathrm{N}, \mathrm{I}, \mathrm{O}, \mathrm{J}$ e Q) foi selecionada uma área (Fig. 2); contendo também os pontos $\mathrm{L}$ e $\mathrm{P}$ que, em conjunto, representam a área mais rica em táxons, mais rica em táxons raros, com maior complementaridade e representatividade. Esta área forma um polígono com os trechos de rios de maior classificação hidrológica. A partir dela foram incluídas na área indicada para conservação as nascentes das microbacias para contemplar o critério de complementaridade. Ao final, foram indicadas as áreas que abrangem as microbacias dos rios Corrente (ponto I), São Bartolomeu (ponto J) e São Mateus (ponto O) (Fig. 2).
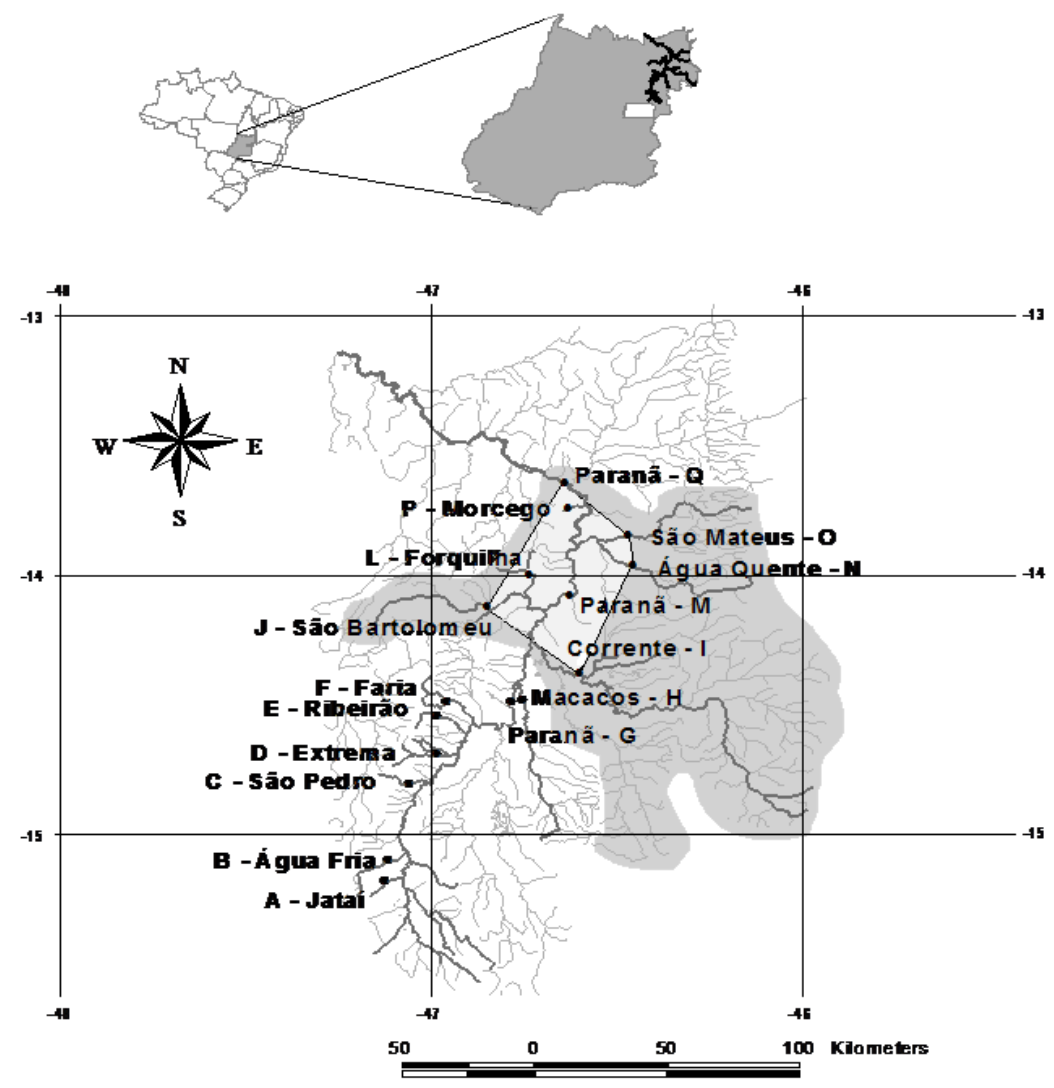

Fig. 2 - Localização dos pontos de coleta de dados e da área proposta para prioridade de conservação de macroinvertebrados bentônicos na bacia hidrográfica do rio Paranã. Os cinco pontos selecionados formam um polígono (cinza claro) de maior representatividade da biota e ao redor (cinza escuro) a área indicada para conservação. 


\section{Discussão}

A bacia do Paranã apresentou dois padrões distintos de similaridade da biota: i- grande similaridade em rios de pequena ordem (nascentes e cabeceiras), independentemente da sua localização geográfica na bacia hidrográfica a montante ou jusante e ii- baixa similaridade entre os pontos de maior ordem hidrológica, localizados na porção média da bacia e que são relativamente próximos geograficamente.

A similaridade em rios de pequena ordem permitiu que o ponto mais representativo deste ambiente (ponto $C$ ) pudesse ser substituído, sem qualquer prejuízo, pelos pontos $\mathrm{P}$ e $\mathrm{F}$ que se encontravam geograficamente mais próximos da área indicada como prioritária para conservação. Além disso, também é plausível que esta biota esteja representada nas nascentes das microbacias dos rios de maior ordem (São Bartolomeu, Corrente e São Mateus), mesmo que estas áreas não tenham sido amostradas, pelo fato de refletirem características hidrológicas semelhantes.

O segundo padrão também deve ser destacado: A baixa similaridade dos pontos na porção média do rio e ao mesmo tempo sua proximidade geográfica. A baixa similaridade da biota indica que estes pontos apresentam características biológicas (táxons, habitats) diferenciadas uns dos outros, 0 que se reflete em uma alta diversidade e de grande interesse para conservação. Já a proximidade desses pontos favorece a indicação de uma área prioritária para conservação na porção média do rio.

A composição diferenciada das espécies entre os pontos de nascente (menor ordem) e de maior ordem seguiu o mesmo padrão descrito na teoria da continuidade fluvial (River Continnum Concept), com a biota de córregos de baixa ordem com composição naturalmente diferenciada da porção média da bacia que possui rios com maior ordem hidrológica (Vannote et al., 1980). Diante destas características é recomendado que a conservação dos macroinvertebrados do Vão do Paranã priorize áreas que contenham tanto os rios de pequenas ordens $\left(1^{a}\right.$ à $\left.3^{a}\right)$, como também rios de ordens intermediárias ( $4^{\mathrm{a}}$ à $6^{\mathrm{a}}$ ) contemplando uma melhor representatividade da biota bentônica. Para garantir a conservação das áreas mais representativas e ricas em informação biológica, propomos que seja considerada de alta prioridade a região média do Rio Paranã. Esta área está destacada no mapa como um polígono central (Fig. 2). Propomos também a conservação das micro-bacias que alimentam estes corpos d'água, área que está destacada no mapa, na cor cinza escuro (Fig. 2).
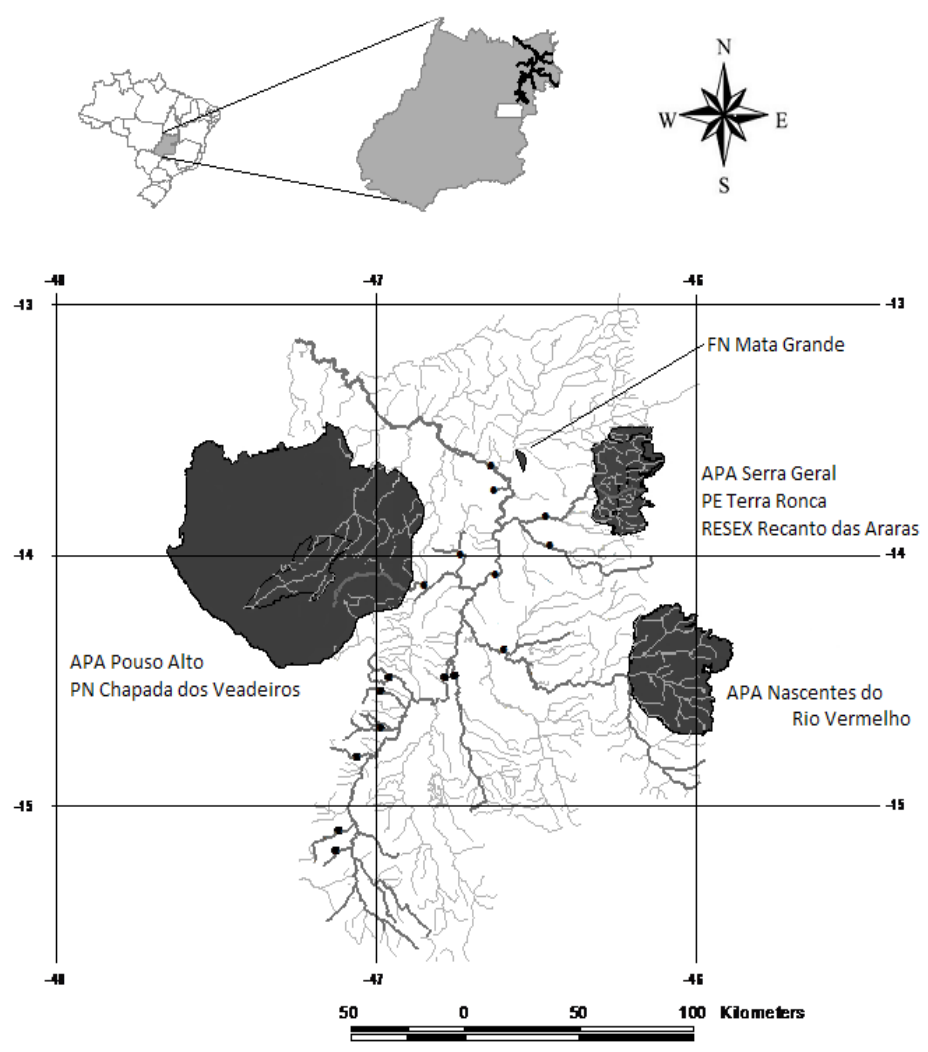

Fig. 3 - Localização das Unidades de Çonservação estaduais e federais presentes no Vão do Rio Paranã, estado de Goiás. Floresta Nacional Mata Funda, Área de Proteção Ambiental (APA) Serra Geral, Parque Estadual Terra Ronca, Reserva Extrativista (RESEX) Recanto das Araras, APA Nascentes do Rio Vermelho, APA Pouso Alto e Parque Nacional da Chapada dos Veadeiros (Fonte: World Wildlife Found. Shape disponível em WWW.observatorio.wwf.org.br). 
Recentemente vêm sendo implantadas Unidades de Conservação (UC) Federais ou Estaduais no Vão do Paranã. Uma delas é a Área de Proteção Ambiental (APA) Nascentes do Rio Vermelho criada no ano de 2001, com uma área de 176.159 hectares (WWF). Esta UC Federal abrange numa pequena parcela das nascentes da bacia do Rio Corrente (Fig. 3). Outras três UCs adjacentes abrangem em conjunto as cabeceiras das microbacias do Rio São Mateus e Água Quente, sendo elas: (i) APA da Serra Geral, (ii) Parque Estadual (PE) Terra Ronca e (iii) Reserva Extrativista (RESEX) Recantos das Araras. A APA de Serra Geral, uma UC Estadual de uso sustentável com área de 49.058 hectares, foi criada no ano de 1996. Esta APA esta sobreposta a uma UC de Proteção Integral, o PE de Terra Ronca com 57.195 hectares criada em 2002 e a RESEX Recanto das Araras de Terra Ronca, UC Federal, com extensão de 11.964 hectares criada em 2006. A oeste esta localizada outra UC, a APA estadual Pouso Alto, com 872.000 hectares, criada em 2001 circundando o Parque Nacional da Capada dos Veadeiros que esta sobreposto a ela. Parte da APA Pouso Alto abrange o Vão do Paranã nas áreas de cabeceira da microbacia dos rios São Bartolomeu e Forquilha. A Floresta Nacional da Mata Grande, de 2.009 hectares, foi criada em 2003 (Fig.3) (WWF).

Estas UCs contribuem principalmente para a manutenção da comunidade de macroinvertebrados bentônicos de cabeceira, que neste trabalho se apresentaram extremamente similares. Apesar de sua grande importância elas não abrangem a região média do rio Paranã deixando desprotegidos os grupos de invertebrados característicos de sistemas aquáticos de maior ordem, justamente a área que estamos apontando como de alta prioridade para conservação. Este fato é particularmente preocupante, pois além de estar desprotegida, a área está localizada na porção média do Paranã que se concentram locais já antropizados da bacia e também áreas com grande tendência de ocupação antrópica futura; tanto por ocupação urbana como agropecuária (Hermuche et al, 2009).

Os resultados contidos neste trabalho fornecem subsídios científicos sobre a estrutura e distribuição da comunidade bentônica na bacia. A união dessas informações associadas à dinâmica de uso da terra no vão do Paranã pode auxiliar uma melhor gestão territorial culminando na meIhor eficiência de conservação das espécies de invertebrados aquáticos da região.

\section{ReferênCIAS}

Araújo, M. 1998. Avaliação da biodiversidade em conservação. Silva Lusitana. 6:19-40.

Arita, H. T. 1993. Rarity in Neotropical bats: correlations with phylogeny, diet, and body mass. Ecological Applications. 3: 506-517.
Barbosa, F.F., Godoy, B.S. \& Oliveira, L.G. 2011. Trichoptera Kirby (Insecta) immature fauna from Rio das Almas Basin and Rio Paranã, Goiás State, Brazil, with new records for some genera. Biota Neotropica. 11:21-25.

Barbour, M.T., Gerritsen, J., Snyder, B.D. \& Stribling,. J.B. 1999. Rapid Bioassessment Protocols for Use in Streams and Wadeable Rivers: Periphyton, Benthic Macroinvertebrates and Fish. 2rd ed. EPA 841-B-99-002. U.S. Environmental Protection Agency, Office of Water, Washington.

Bispo, P.C., Oliveira, L.G., Crisci, V.L. \& Silva, M.M. 2001. A pluviosidade como fator de alteração da entomofauna bentônica (Ephemeroptera, Plecoptera e Trichoptera) em córregos do planalto Central do Brasil. Acta Limnologica Brasiliensis. 13: 1-9.

Brandon, K., da Fonseca, G.A.B., Rylands, A.B. \& Danni, J.C.M.. 2005. Challenges and opportunities in Brazilian conservation. Conservation Biology. 19:595-600.

Buckley, G.P. \& Forbes, J.E. 1978. Ecological evaluation using biological habitats an appraisal. Landscape Planning. 5:263-280.

Buss, D.F., Baptista, D.F. \& Nessimian, J.L. 2003. Bases conceituais para a aplicação de biomonitoramento em programas de avaliação da qualidade da água de rios. Caderno Saúde Pública. 19:495-473.

Cousins, S.H. 1991. Species diversity measurement: choosing the right index. Trends in Ecology \& Evolution. 6:190-193.

De Marco P. \& Vianna D.M. 2005. Distribuição do esforço de coleta de Odonata no Brasil: subsídios para escolha de áreas prioritárias para levantamentos faunísticos. Lundiana. 6:13-26.

Diniz-Filho, J.A.F., De Marco, P. \& Hawkins, B.A. 2010. Defying the curse of ignorance: perspectives in insect macroecology and conservation biogeography. Insect Conservation and Diversity. 3:172-179.

Dominguez, E., Hubbard, M.D., Pescador, M.L. \& Molineri, C. 2001. Ephemeroptera, p. 17-53. In Fernández, H. R. \& Dominguez, E. (eds.). Guía para la determinación de los artrópodos bentónicos sudamericanos. Tucuman: Universidad Nacional de Tucumán.

Dudgeon, D. 2008. Aquatic ecosystems: tropical stream ecology. Elsevier Science, London.

Dutra, S.L. 2006. Avaliação da biodiversidade bentônica no Vale do Paranã (GO), visan- 
do a identificação de áreas prioritárias para conservação. Dissertação Universidade de Brasília. Brasília.

Ferreira Jr., L.G. 2008. A encruzilhada socioambiental - biodiversidade, economia e sustentabilidade no cerrado. 1rd ed. Goiânia: Editora UFG.

Gaston, K. 1996. What is biodiversity?, p. 1-9. In Gaston, K. (ed.). Biodiversity - A Biology of Numbers and Difference. UK: Blakwell Science.

Harold, A.S.\& Randall, D.M. 1994. Areas of Endemism: Definition and Recognition Criteria. Systematic Biology. 43:261-266.

Hermuche, P.M., Ferreira, N.C. \& Sano, E.E. 2009. Proposta metodológica para elaboração de mapa de tendência de uso da terra no Vão do Paranã, Goiás, baseada em condicionantes ambientais e sócio-econômicos. Anais XIV Simpósio Brasileiro de Sensoriamento Remoto. 5873-5880.

Junqueira,M.V.,Amarante,M.C., França,E.S. \& Dia,C.F.S. 2000. Biomonitoramento da qualidade das águas da bacia do Alto Rio das Velhas (MG/Brasil) através de macroinvertebrados. Acta Limnologica Brasiliensia.12:73-87.

Kareiva, P. \& Marvier, M. 2003. Conserving biodiversity coldspots. American Scientist. 5:344-351.

Karr, J.R. \& Chu,E.W. 1999. Restoring life in running waters. Washington D. C: Island Press.

Lambers,H., Chapin,I. \& Pons,T.L. 1998. Plant physiological ecology. Springer-Verlag, NewYork.

Lewinsohn,T.M., Freitas,A.V.L. \& Prado,P.I. 2005. Conservação de invertebrados terrestres e seus habitats no Brasil. Megadiversidade. 1: 62-69.

Linke,S., Pressey,R.L., Bailey,R.C. \& Norris, R.H. 2007. Management options for river conservation planning: condition and conservation re-visited. Freshwater Biology. 52: 918-938.

Lomolino, M.V. \& Perault, D.R. 2004 Geographic gradients of deforestation and mammalian communities in a fragmented, temperate rain forest landscape. Global Ecology and Biogeography. 13:55-64.

Looy, K.V., Honnay, O., Pedroli, B. \& Muller, S. 2006. Order and disorder in the river continuum: the contribution of continuity and connectivity to floodplain meadow biodiversity. 33:1615-1627.
Lortie, C.J., Brooker, R.W., Choler, P., Kikvidze, Z., Michalet, R., Pugnaire, F.I. \& Callaway, R.M., 2004. Rethinking plant community theory. Oikos. 107: 433-438.

Martins-Silva,M.J., Engel,D.W., Da Rocha,F.M. \& Araújo,J. 2008. Trichoptera imatures in Paranã river basin, Goiás state, with new records for genera. Neotropical Entomology 37:735-738.

McGeoch, M.A. 1998. The selection, testing and application of terrestrial insects as bioindicators. Biological Reviews 73:181-201.

Merritt, R. W. \& K. W. Cummins. 1988. An introduction to the aquatic insect of North America. 2 ed. Dubuque, Kendal/ Hunt Publishing.

Ministério do Meio Ambiente (MMA). 1999. Pró-Natureza/Funatura, Conservation International, Fundação Biodiversitas \& Universidade De Brasília. Avaliação e Ações Prioritárias para a Conservação da Biodiversidade do Cerrado e Pantanal. Brasília.

Miserendino, M. L. \& Pizzolón, L. A. 2001. Rapid assessment of river water quality using macroinvertebrates: A family level biotic index for the patagonic andean zone. Acta Limnologica Brasiliensia. 11:137-148.

Mora,C., Tittensor,D.P., Adl,S., Simpson,A.G.B. \& Worm,B. 2011. How Many Species Are There on Earth and in the Ocean? PLoS Biology. 9.

Myers, N. 1988. Treatened biomas: "hot-spots" in tropical rain forest. Environmentalist. 10:243-256.

Myers, N. 1990. The biodiversity challenge: expanded hot-spots analysis. Environmentalist n.64 p.165-169.

Myers, N., Mittermeier, R.A., Mittermeier,C.G., Da Fonseca, G.A.B. \& Kent, J. 2000. Biodiversity hotspots for conservation priorities. Nature.403:853-858.

Nimer, E. 1979. Climatologia do Brasil. Superintendência de Recursos Naturais e Meio Ambiente (SUPREN), IBGE, Rio de Janeiro.

Pringle,C.M. 2001. Hydrologic Connectivity and the Manegement of Biological Reseves: a Global Perspective. Ecological Applications.11: 981-998.

Rosenberg,D.M. \& Resh,V.H. 1993. Freshwater Biomonitoring and Benthic Macroinvertebrates. Chapman \& Hall, London.

Silveira,M.P., Baptista,D.F., Buss,D.F. \& Nessimian,J.L. 2005. Application of biolog- 
ical measures for stream integrity assessment in slouth-east Brazil. Environmental Monitoring and Assessment. 101:117-128.

Strahler,H.N. 1957. Quantitative analysis of watershed geomorphology. American Geophysics Union Transactions. 3:913-920.

Strayer,D.L. 2006. Challenges for freshwater invertebrate conservation. Journal of the North American Benthological Society. 25:271-287.

Ulrich,W. 2008. Species abundance distributions in space and time. Ecological Questions. 9:15-20.

Vane-Wright,R.I., Humphries,C.J. \& Williams,P.H. 1991. What to protect? systematics and the agony of choice. Biological Conservation. 235-254.
Vannote,R.L., Minshall,G.W., Cummins,K.W., Sedell,J.R. \& Cushing,C.E. 1980. River Continuum Concept. Canadian Journal of Fisheries and Aquatic Sciences.37:130-137.

Wiggins,G.B. 1977. Larvas of north american caddisfly genera Trichoptera. University of Toronto Press, Toronto.

World Wildlife Found (WWF). Observatório de UCs, Shape disponível em www.observatorio.wwf.org.br (último acesso em 20/01/2013).

Zaher,H. \& Young,P.S. 2003. As coleções zoológicas brasileiras: panorama e desafios. Ciência e Cultura. 55:24-26.

Recebido em 08.II.2012 Aceito em 26.II.2013 